\title{
Roles of Microembolus and Plasma D-dimer in the Evaluation of Warfarin Anticoagulant Therapy Efficacies for Patients with Atrial Fibrillation
}

\author{
Yun Xu, Bing Wang, Li-Jiao Jiang, Xiao-Ping Lu, Xiao-Ying Zhao, Fang Wang \\ Department of Cardiology, Wuzhong People's Hospital, Suzhou 215128, China
}

\section{ABSTRACT}

Background: To investigate the roles of microemboli and plasma D-dimer in the evaluation of warfarin anticoagulant therapy efficacies for patients with atrial fibrillation (AF).

Methods: Fifty-six patients with AF were treated with aspirin antiplatelet therapy (Group ASP), and 40 patients with AF were treated with warfarin anticoagulant therapy (Group WAR). Microemboli and plasma D-dimer in these two groups were monitored and compared before and after treatment.

Results: Group ASP had 21 and 17 patients test positive for microemboli before and after treatment, respectively. Also, there was no significant difference in the rate of microembolus detection before or after treatment. Group WAR had 14 and five patients test positive for microemboli before and after treatment, respectively; the rate of microembolus detection was significantly reduced after treatment. The levels of plasma D-dimer in the ASP and WAR groups were significantly reduced after treatment $(327 \pm 73 \mu \mathrm{g} / \mathrm{L}$ versus $235 \pm 61$ $\mu \mathrm{g} / \mathrm{L}$ and $313 \pm 81 \mu \mathrm{g} / \mathrm{L}$ versus $170 \pm 67 \mu \mathrm{g} / \mathrm{L}$, respectively, $P<.05)$, with a greater reduction in Group WAR.

Conclusions: Microemboli and D-dimer can be used as indicators in the evaluation of both embolism risk and therapeutic efficacies in patients with AF.

\section{INTRODUCTION}

Atrial fibrillation (AF) is the most prevalent cardiac arrhythmia in the general population and is associated with a high risk of developing morbidities, such as hemodynamic instability, thromboembolism, stroke, hospital readmission, and increased health care costs [Macle 2016]. AF is linked to a five-fold increase in the risk of cerebrovascular events and is related to approximately $20 \%$ of stroke events [Albertsen 2013].

The presence of congestive heart failure, hypertension, advanced age, diabetes mellitus, and use of the stroke (CHADS2) scoring system, which are clinical factors that are easy for physicians to remember and apply, have been widely

Received September 8, 2018; received in revised form November 11, 2018; accepted November 14, 2018.

Correspondence: Yun Xu, Department of Cardiology, Wuzhong People's Hospital, Suzhou 215128, China; +86-512-33333600; fax: +86-51268560200 (e-mail: docxuyun6688@126.com). validated for the risk stratification of stroke in patients with non-valvular atrial fibrillation (NVAF) [Gage 2001].

The current guidelines recommend anticoagulant therapy for patients with a CHADS2 score $\geq 2$ points, which is due to the risk of ischemic stroke out-weighing the risk of bleeding that is associated with anticoagulant therapy [European Heart Rhythm Association 2010; You 2012; January 2014]. However, thromboembolism can occur in patients with $\mathrm{AF}$ who have a low CHADS2 score (CHADS2 score $=0$ or 1 point) [Gage 2001]

Therefore, finding simple and objective indicators to facilitate the prediction of embolism risk in patients with $\mathrm{AF}$, especially for screening the patients with a high AF risk so as to guide their individual anticoagulant therapy, will have important practical significance.

Because patients with $\mathrm{AF}$ exist in a pre-thromboembolic state, many studies have confirmed that determining the plasma D-dimer level, combined with clinical risk factors, can effectively predict thromboembolic events in NVAF patients [Sadanaga 2010; Erdogan 2014; El Borgi 2015; Kim 2016]. However, a single micro-embolic signal does not necessarily lead to the clinical symptoms of a cerebral embolism [Nagy-Baló 2017].

In recent years, the application of Transcranial Doppler (TCD) to monitor microemboli has been more frequently used as a method to determine the risk of cardiogenic embolism [Sauren 2009; Nagy-Balo 2014; Seguchi 2015]. However, whether microemboli can act as an independent or additional indicator of embolism risk in NVAF patients requires further study.

We applied antiplatelet and anticoagulant therapies to two groups of patients with $\mathrm{AF}$ and compared the changes of plasma D-dimer and microemboli in these two groups before and after treatment. The aim of this study was to identify whether plasma D-dimer and microembolus can be used to assess the embolism risk and to evaluate the therapeutic effects in patients with AF by comparing these two indices and their correlations with the embolism risk of AF.

\section{MATERIALS AND METHODS}

Study subjects: Ninety-six patients with AF, who were treated in our hospital from March 2013 to Nov. 2016, were enrolled in this study. The patients were 63 males and 33 females; the patients were aged 55 years to 86 years, with a median age of 68 years. 
Table 1. Patients' Characteristics in Group ASP and WAR

\begin{tabular}{lccc}
\hline & Group WAR $(\mathrm{N}=40)$ & Group ASP $(\mathrm{N}=56)$ & $P$ \\
\hline Age $( \pm \mathrm{SD})$ & $66.3 \pm 8.0$ & $68.0 \pm 9.3$ & .77 \\
Male $(\mathrm{N})$ & 28 & 35 & .55 \\
LAD $(\mathrm{mm})$ & $41.0 \pm 6.2$ & $42.1 \pm 5.5$ & .76 \\
$\operatorname{LVEF}(\%)$ & $58.9 \pm 11.0$ & $60.0 \pm 12.1$ & .85 \\
CHADS2 score & $3.3 \pm 1.1$ & $3.1 \pm 0.9$ & .49
\end{tabular}

$\mathrm{AF}$ in all of the patients was confirmed by an electrocardiogram, and their AF symptoms lasted from one week to 11 years. The CHADS2 scores in these patients ranged from 2 points to 6 points [Gage 2001], with an average of $3.0 \pm 1.2$ points.

Forty patients, who were free from warfarin contraindications and who signed the informed consent for the application of warfarin anticoagulant therapy, were grouped into Group WAR with the international normalized ratio (INR) target of warfarin treatment between 1.6 and 2.5.

The remaining 56 patients only agreed to the aspirin antiplatelet therapy (100 mg, once daily) and were grouped into Group ASP.

There was no significant difference in the age, sex, or CHADS2 scores between the two groups (Table 1). The study protocol was approved by the Human Research Ethics Committee of Wuzhong People's Hospital.

We ensured that all of the enrolled patients were clear of the following complications: Heart valve disease, congenital heart disease, pulmonary heart disease, pericarditis, thyroid dysfunction, stroke or transient ischemic attack $\leq$ one month, deep vein thrombosis, pulmonary embolism, and blood diseases. All patients were confirmed to have no cardiac thrombosis by a transthoracic ultrasonic cardiogram (UCG).

\section{Procedure}

Each patient was monitored for microemboli and their plasma D-dimer levels were tested before and one month after aspirin antiplatelet therapy (Group ASP), or before and when INR stability was achieved after warfarin anticoagulant therapy. The changes between the two groups were compared before and after treatment.

\section{Monitoring Microemboli}

One Embo-Dop type transcranial doppler instrument (TCD) (DWL, Germany) was used. The diagnostic criteria of microemboli [Basic identification criteria of Doppler microembolic signals 1995] were a sharp bird or whistle sound that appeared in the frequency spectrum of blood flow, with a short time course $(<300 \mathrm{~ms}), \mathrm{S} / \mathrm{N}$ ratio $>3 \mathrm{~dB}$, and single direction. The specific diagnostic methods were as follows: 1) Applied the probe to routinely detect the internal and external cranial blood vessels with a frequency of $4 \mathrm{MHz}$ and $2 \mathrm{MHz}$, respectively, followed by a monitoring for microemboli in the bilateral middle cerebral arteries; 2) fixed the probe in the temporal window and after the middle cerebral artery using one frisket, and then started the monitoring at
Table 2. Positive MES and D-dimer Level Before and After Treatment

\begin{tabular}{ccccc}
\hline & \multicolumn{2}{c}{ Group ASP $(\mathrm{N}=56)$} & \multicolumn{2}{c}{ Group WAR $(\mathrm{N}=40)$} \\
\hline & Before & After & Before & After \\
D-dimer $(\mu \mathrm{g} / \mathrm{L})$ & $327 \pm 73$ & $235 \pm 61^{*} \dagger$ & $313 \pm 81$ & $170 \pm 67^{*} \dagger$ \\
Positive MES $(\mathrm{n})$ & 21 & $17+$ & 14 & $5 *+$ \\
\hline
\end{tabular}

Comparison in Group ASP and Group WAR before and after treament: * $P$ $<.05$; comparision between Group ASP and Group WAR before and after treament: $\nmid P<.05$.

the beginning or distal end of the stenosis of the middle cerebral artery; 3) and the experienced TCD physicians closely observed the spectral changes during the monitoring (monitoring time was $30 \mathrm{~min}$.). The result was defined as positive if microemboli were detected or negative if no microemboli were detected.

\section{Detection of D-dimer}

A total of $3 \mathrm{~mL}$ fasting venous blood was collected in the early morning, and the blood was centrifuged to separate the plasma according to the requirements for the detection of the D-dimer level using the immunoturbidimetry method (Hitachi 7600 automatic Biochemical analyzer and D-dimer assay kits, SEKISUI Co, Ltd., Japan). All the testing items met the quality control criteria.

\section{Statistical Analysis}

SPSS15.0 statistical software was used for the analysis. The data were expressed as $\overline{\mathrm{x}} \pm \mathrm{s}$. The $\mathrm{D}$-dimer levels before and after treatment were compared with the paired t-test. The intergroup comparison used the independent-sample t-test. The count data were expressed as the number of patients, and the positive microemboli rate before and after treatment and between the two groups were compared with the chi-square test. A $P$ value $<0.05$ was considered statistically significant.

\section{RESULTS}

Aspirin antiplatelet therapy: Before treatment, Group ASP had 21 patients test positive for microemboli and 35 patients test negative for microemboli. After treatment, Group ASP had 17 patients test positive for microemboli and 39 patients test negative for microemboli. The plasma $\mathrm{D}$-dimer levels were $327 \pm 73 \mu \mathrm{g} / \mathrm{L}$ and $235 \pm 61 \mu \mathrm{g} / \mathrm{L}$ before and after treatment, respectively (Table 2).

Warfarin anticoagulant therapy: Before treatment, Group WAR had 14 patients test positive for microemboli and 26 patients test negative for microemboli. After treatment, Group WAR had five patients test positive for microemboli and 35 patients test negative for microemboli. The plasma D-dimer levels were $313 \pm 81 \mu \mathrm{g} / \mathrm{L}$ and $170 \pm 67 \mu \mathrm{g} / \mathrm{L}$ before and after treatment, respectively (Table 2). 
DISCUSSION

The most commonly recommended anticoagulant for the prevention of thrombosis in patients with $\mathrm{AF}$ is warfarin, but patients who are reluctant to initiate an anticoagulant therapy are prescribed aspirin antiplatelet therapy [Wyse 2002; Hart 2007].

We observed that warfarin anticoagulant therapy reduced patients' plasma D-dimer levels and positivity rate of microemboli. Conversely, aspirin antiplatelet therapy only mildly reduced patients' plasma D-dimer levels and had no effect on microemboli.

Patients with AF experience an activation of the coagulant and fibrinolytic systems. The risk of stroke in patients with $\mathrm{AF}$ increases as the D-dimer level increases [Krarup 2011; Matsumoto 2013].

Multivariate analysis has revealed that non-paroxysmal atrial fibrillation, congestive heart failure, recent embolism history, and plasma D-dimer level are the independent predictors of left atrial thrombosis by transesophageal UCG, among which the strongest predictor is the plasma $\mathrm{D}$-dimer level [Habara 2007]. The observation that microemboli have been simultaneously monitored in the bilateral middle cerebral arteries suggests that cardiogenic emboli have a higher specificity. In addition, less paroxysmal microemboli and asymptomatic isolated $\mathrm{AF}$, when compared with chronic and symptomatic isolated $\mathrm{AF}$ cases with more microemboli, are a type of benign arrhythmia [Go 2003]. The number of microemboli is closely related to the micro-cerebral ischemic foci detected by cerebral magnetic resonance imaging.

In recent years, an increasing number of studies have used microembolus monitoring to predict the risk of cardiogenic embolism, and this confirms the clinical feasibility of this method in patients with AF [Sauren 2009; Nagy-Balo 2014; Seguchi 2015].

At present, clinical factors are the basis for guiding antithrombotic therapy in patients with $\mathrm{AF}$, and CHADS2 scores exhibit a significantly positive correlation with the incidence rate of thrombosis in these patients [Gage 2001]. Warfarin can significantly reduce the risk of embolism in high-risk patients with AF. A number of studies have confirmed that the effect of aspirin antiplatelet therapy on the reduction of emboli in patients with AF is significantly weaker than warfarin, especially in the patients with a higher embolism risk [Wyse 2002; Hart 2007].

The embolism risk in Group ASP and Group WAR was decreased after treatment, and the in vivo D-dimer levels, which reflect the coagulant and fibrinolytic activities, were simultaneously decreased. Because warfarin has a significantly better preventive effect against thromboembolism than aspirin, the D-dimer level in Group WAR was more significantly decreased, and this result is consistent with previous studies. Therefore, this study supports the use of the D-dimer level as a predictive indicator for embolism risk and antithrombotic efficacy evaluation in patients with AF [Sadanaga 2010; Erdogan 2014; Kim 2016].

We observed that Group ASP had 21 and 17 patients test positive for microemboli before and after treatment, respectively, which suggests there was no significant reduction in microemboli after treatment $\left(37.5 \%\right.$ versus $30.4 \%, \chi^{2}=0.64$, $P=.42)$. Conversely, Group WAR had 14 and 5 patients test positive for microemboli before and after treatment, respectively, which reveals a significant reduction in microemboli after treatment $\left(35.0 \%\right.$ versus $\left.12.5 \%, \chi^{2}=5.59, P<.05\right)$.

In recent years, the effect of aspirin in treating patients with both $\mathrm{AF}$ and a high risk of embolism has been questioned, and its status has been significantly reduced [Melanie 2013]. We observed that aspirin did not reduce the microembolus detection rate, and this result seems to support the above conclusions. However, warfarin significantly reduced the microembolus detection rate, and therefore significantly reduced the embolism risk. Therefore, these results would indicate that microemboli can directly and better reflect the embolism risk in patients with $\mathrm{AF}$. We believe that monitoring microemboli is clinically simple and feasible, can be used as a supplement to the CHADS2 scoring system, and can exhibit important clinical application values in guiding anticoagulant therapy in patients with a high embolism risk [Held 2015].

The D-dimer level can be influenced by many factors including coagulant and fibrinolytic dysfunctions in other body parts. Therefore, the D-dimer level may not accurately reflect the embolism risk in the left atrial appendage. This study did not quantify the monitored microemboli and did not rule out the possibility that some microembolus signals were generated by gas and other small solid particles. However, we believe that the combination of the D-dimer level and monitored microemboli can be effectively used to assess embolism risk in specific patients with $\mathrm{AF}$.

This study had several limitations. The number of patients was small, and the clinical embolism events were not used as a hard end point to observe their relevance with the D-dimer level and microemboli due to our research time limitation. To further examine the significance of D-dimer and microemboli for the prediction of embolism risk in patients with $\mathrm{AF}$, we propose the following for future studies: Divide the patients with $\mathrm{AF}$ into different subgroups according to whether their D-dimer levels are increased or the microemboli are positive or negative, and give different antithrombotic measures so as to prospectively observe the incidence of thromboembolic events.

\section{REFERENCES}

Albertsen IE, Rasmussen LH, Overvad TF, Graungaard T, Larsen TB, Lip GY. 2013. Risk of stroke or systemic embolism in atrial fibrillation patients treated with warfarin: a systematic review and meta-analysis. Stroke 44:1329-36.

Basic identification criteria of Doppler microembolic signals. 1995. Consensus Committee of the Ninth International Cerebral Hemodynamic Symposium. Stroke 26:1123.

El Borgi W, Romdhane S, Sdiri W, Longo S, Hafsia R, Boujnah MR. 2015. Measurement of d-dimers in nonvalvular atrial fibrillation. First prospective Tunisian study. Ann Cardiol Angeiol (Paris) 64:279-84.

Erdogan D, Uysal BA, Aksoy F, et al. 2014. Strict heart rate control attenuates pro-thrombotic state and platelet activity in patients with non-valvular permanent atrial fibrillation. Clin Hemorheol Microcirc 56:219-29. 
European Heart Rhythm Association; European Association for CardioThoracic Surgery, Camm AJ, et al. 2010. Guidelines for the management of atrial fibrillation: the task force for the management of atrial fibrillation of the European Society of Cardiology. Eur Heart J 31:2369-429.

Gage BF, Waterman AD, Shannon W, Boechler M, Rich MW, Radford MJ. 2001. Validation of clinical classification schemes for predicting stroke: results from the National Registry of Atrial Fibrillation. JAMA 285:2864-70.

Go AS, Hylek EM, Chang Y, et al. 2003. Anticoagulation therapy for stroke prevention in atrial fibrillation: how well do randomized trials translate into clinical practice? JAMA 290:2685-92.

Habara S, Dote K, Kato M, et al. 2007. Prediction of left atrial appendage thrombi in non-valvular atrial fibrillation. Eur Heart J 28:2217-22.

Hart RG, Pearce LA, Aguilar MI. 2007. Meta-analysis: antithrombotic therapy to prevent stroke in patients who have nonvalvular atrial fibrillation. Ann Intern Med 146:857-67.

Held C, Hylek EM, Alexander JH, et al. 2015. Clinical outcomes and management associated with major bleeding in patients with atrial fibrillation treated with apixaban or warfarin: insights from the ARISTOTLE trial. Eur Heart J 36:1264-72.

January CT, Wann LS, Alpert JS, et al. 2014. 2014 AHA/ACC/HRS guideline for the management of patients with atrial fibrillation: a report of the American College of Cardiology/American Heart Association Task Force on Practice Guidelines and the Heart Rhythm Society. J Am Coll Cardiol 64:e1-76.

Kim TW, Song IU, Chung SW, Kim JS, Koo J, Lee KS. 2016. Serum D-dimer Levels Are Proportionally Associated with Left Atrial Enlargement in Patients with an Acute Ischemic Stroke due to Non-valvular Atrial Fibrillation. Intern Med 55:1447-52.

Krarup LH, Sandset EC, Sandset PM, Berge E. 2011. D-dimer levels and stroke progression in patients with acute ischemic stroke and atrial fibrillation. Acta Neurol Scand 124:40-4.

Macle L, Cairns J, Leblanc K, et al. 2016. 2016 Focused Update of the
Canadian Cardiovascular Society Guidelines for the Management of Atrial Fibrillation. Can J Cardiol 32:1170-85.

Matsumoto M, Sakaguchi M, Okazaki S, et al. 2013. Relationship between plasma (D)-dimer level and cerebral infarction volume in patients with nonvalvular atrial fibrillation. Cerebrovasc Dis 35:64-72.

Nagy-Balo E, Kiss A, Condie C, Stewart M, Edes I, Csanadi Z. 2014. Predictors of cerebral microembolization during phased radiofrequency ablation of atrial fibrillation: role of the ongoing rhythm and the site of energy delivery. Pacing Clin Electrophysiol 37:1436-41.

Nagy-Baló E, Martirosyan M, Sándorfi G, et al. 2017. Cerebral microembolization during pulmonary vein isolation: relation to post-ablation silent cerebral ischemia. Cardiol J 24:234-41.

Sadanaga T, Kohsaka S, Ogawa S. 2010. D-Dimer Levels in Combination with Clinical Risk Factors Can Effectively Predict Subsequent Thromboembolic Events in Patients with Atrial Fibrillation during Oral Anticoagulant Therapy. Cardiology 117:31-6.

Sauren LD, la Meir M, de Roy L, et al. 2009. Increased number of cerebral emboli during percutaneous endocardial pulmonary vein isolation versus a thoracoseopic epicardial approach. Eur J Cardiothorac Surg 36:833-7.

Seguchi O, Saito K, Fukuma K, et al. 2015. Evaluation of micro-emboli in a patient with ventricular assist device support with hemolysis. J Artif Organs 18:276-9.

Wyld ML, Clayton PA, Morton RL, Chadban SJ. 2013. Anti-coagulation, anti-platelets or no therapy in haemodialysis patients with atrial fibrillation: A decision analysis. Nephrology (Carlton) 18:783-9.

Wyse DG, Waldo AL, DiMarco JP, et al. 2002. A comparison of rate control and rhythm control in patients with atrial fibrillation. N Engl J Med 347:1825-33.

You JJ, Singer DE, Howard PA, et al. 2012. Antithrombotic Therapy for Atrial Fibrillation: Antithrombotic Therapy and prevention of Thrombosis, 9th ed: American College of Chest Physicians Evidence-Based Clinical Practice Guidelines. Chest 141:e531S-75S. 